\title{
Premature Rupture of Membrane - A Clinical Study In Comilla Medical College Hospital
}

\author{
SHAHELA NAZNEEN ${ }^{1}$, FARHANA BEGUM ${ }^{2}$, SHAKILA NARGIS ${ }^{3}$
}

\begin{abstract}
:
Objective: To find out the sociodemographic characteristics, complications of pregnant woman with premature rupture of membrane (PROM) and asses the outcome of it.

Materials and Methods: This prospective study was carried out at the department of Obstetrics \& gynaecology of Comilla Medical College Hospital (CoMCH) from January 2009 to December 2009. All pregnant women admitted with PROM during this period were taken as study population. After taking proper history they were categorized as term or preterm PROM on the basis of duration of gestation estimated from $1^{\text {st }}$ day of last menstrual period (LMP), previous antenatal records, clinical examination \& also previous ultrasonography (USG) reports. Out of sociodemographic characteristics age distribution and gravidity were noted. Diagnosis was done on the basis of examination and investigations. All the women with PROM were then followed up, management was given according to protocol and caesarean section was performed according to indication. Birth weight and Apgar score of newborns were noted after delivery.

Results: There were 198 cases of PROM recorded among 3142 admitted obstetrical patients over one year period. The hospital incidence of PROM was found to be $6.3 \%$. Among them, $57.6 \%$ patients were admitted at term and $42.4 \%$ patients came before 37 completed weeks of gestation. Most of the pregnant women were between $20-24$ years of age (54\%), 41\% were primigravida (59\%) were multigravida. About 48.5\% women presented with different complications associated with PROM. Among which $15.7 \%$ patients had oligohydramnios, $8.5 \%$ patients presented with chorioamnionitis suggested by culture report of high vaginal swab. Urine for culture and sensitivity was also done. About five (5.2\%) developed premature labour before 37 th week of gestation. About 10\% women developed obstetric complications (failed trial) associated with medical diseases. Seventy seven patients were delivered by caesarean section, $16.2 \%$ vaginally, $4.04 \%$ patients responded to conservative management and $2.5 \%$ patients were referred to tertiary centre for extreme prematurity with associated complicating factors. Most of the babies (38.4\%) were born with birth weight between 2.1$2.5 \mathrm{~kg}$ and $10.3 \%$ babies were less than $1.5 \mathrm{~kg}$.

Conclusion: Term PROM was more in comparison to PPROM and most of them were multigravida. Caesarean section rate was high. Most common complication of was subclinical urogenital infection (53\%) next oligohydramnios (25\%) \& (13.5\%) women presented with chorioamnionitis.
\end{abstract}

Key words: Premature rupture of membrane, chorioamnionitis, Prematurity.

\section{Introduction:}

Premature rupture of membrane (PROM) is a syndrome characterized by spontaneous rupture of the chorioamnion before the onset of uterine contractions $^{1}$. It occurs in approximately $10 \%$ of all pregnancies $^{2}$. The membranes may rupture either at term, that is after 37 completed gestational weeks, or before term, when it is called preterm prelabour rupture of membranes (PPROM). In these cases there is serious concern regarding the obstetric complications as well as perinatal morbidity and mortality ${ }^{1}$.

1. Assistant Professor, Department of Obstetrics \& Gynaecology, Comilla Medical College, Comilla.

2. Junior Consultant (Gynae), Upa-zilla Health Complex, Nangalkort, Comilla.

3. Consultant Gynaecologist, Sheba Hospital (Pvt) Ltd, Comilla. 
In majority of the condition causes is not known ${ }^{3}$. Theoretically, PROM may occur because of increased friability of the membranes, decreased tensile strength of the membrane or an increase in intrauterine pressure or both ${ }^{1-3}$.

Under normal circumstances, the tensile strength of the membranes increases until 20 weeks and then plateaus until 39 week when it starts to decrease dramatically. The amniotic membranes are connective tissue structure and their tensile strength depends on the synthesis, degradation and quality of their collagen ${ }^{2}$. An abnormal collagen structure may be responsible for $\mathrm{PROM}$ as evidenced by the high frequency of PROM in women affected by connective tissue disorders such as the Ehlers-Danlos syndromeu†. Also, tobacco smoking and nutritional deficiencies of zinc, copper and ascorbic acid cause abnormal collagen cross-linking and may produce $\mathrm{PROM}^{2}$.

Intra-amniotic infection may cause alterations in the tensile strength of the fetal membranes by mechanisms different than those involved in the increase in collagenolytic activity. Several of the microorganisms found in women with PPROM and amniotic infection produce proteolytic enzymes that can weaken the fetal membranes. Furthermore, the host inflammatory response to intra-amniotic infection almost certainly plays a role in the mechanism of PPROM. This inflammatory response includes the production of cytokines that stimulate prostaglandin production by the amnion and chorion. Prostaglandins stimulate uterine contractility and cause increased collagen degradation. Also, the glucocorticoids produced in response to the stress of the intra-amniotic infection may be involved in facilitating rupture of the membranes ${ }^{2}$.

PROM is a significant occurrence as it can cause maternal complications, increased operative procedures and perinatal morbidity and mortality. The obstetrician is often in a dilemma regarding the future course of action and a careful consideration of factors like assessment of the gestational age, likelihood of infection and availability of neonatal intensive care facilities helps to decide the appropriate management ${ }^{1}$.

Infection is closely associated either as an etiologic factor or as a consequence of PROM. From the maternal point of view, chorioamnionitis is a major problem which can lead to intrapartum and postpartum sepsis and rarely, septicemia.
Fetal deformities as a consequence of longstanding oligohydramnios and neonatal morbidity and mortality due to sepsis and prematurity are the important neonatal problems in $\mathrm{PROM}^{1}$.

PROM is very often seen in a busy obstetric ward in our country. Proper diagnosis, close monitoring of the patients, appropriate antimicrobial therapy can improve the maternal and fetal outcome.

\section{Materials \& Methods:}

This prospective study was carried out at the Department of Obstetrics \& Gynaecology of Comilla Medical College Hospital (CoMCH) from January 2009 to December 2009. From all admitted pregnant patients, only cases of Premature Rupture of Membrane (PROM) were taken for the study.

After formulation of aim of the study, a clinical data sheet was made for recording all informations of the pregnant women. A verbal informed consent was taken from each woman. After history taking, duration of gestational age was estimated from $1^{\text {st }}$ day of last menstrual period (LMP), previous antenatal records, clinical examination \& also previous ultrasonography (USG) reports.

Diagnosis of PROM was confirmed from history of gush of fluid coming from the vagina, demonstration of amniotic fluid leakage from the cervix by a sterile speculum examination (P/S) or pooling of amniotic fluid in posterior vaginal fornix and observation of oligohydramnios by amniotic fluid index (AFI) through USG. During P/S examination, a high vaginal \& endocervical swab was taken from all the patients and sent for culture \& sensitivity, a blood sample for leukocyte count and urine for routine examination \& culture sensitivity(C/S) was sent. E.coli was most common pathogen.

Plan of management of patients with PROM was decided according to the condition of the patient, duration of gestational age, duration of membrane rupture, associated any complicating factors, maternal \& fetal condition and also neonatal intensive care facility. Prophylactic antibiotic (erythromycin base $250 \mathrm{mg}$ by mouth four times per day for 7 days plus (+) amoxicillin 500mg by mouth three times per day for 7 days) was given to reduce maternal and neonatal infective morbidity and to delay delivery. If the membranes had been ruptured for more than 18 hours, injection ampicillin 2gm IV every 6 hourly given to help reduce Group B streptococcus infection in the neonate. 
Dexamethasone injection 12mg IM, two doses 12 hours apart was given to the mother to improve fetal lung maturity if duration of pregnancy was less than 34 weeks.

Conservative management was given when gestational age less than 37 weeks. But PROM with more than 37 weeks, a vaginal examination (P/V) was done to assess Bishop's score. After initial evaluation, P/V was restricted to minimum and a progress of labour was monitored from nature of uterine contraction \& descent of fetal head. Delivery was augmented either by medical induction or Lower Uterine Caesarean section(LUCS). C/S was performed when PROM was complicated with other factors.

After delivery all the babies were assessed by Apgar score and sent to neonatology dept if any complicating factor was present.

\section{Results:}

A total 198 cases of Premature Rupture of Membrane (PROM) were recorded from January 2009 to December 2009 among 3142 admitted pregnant patients in $\mathrm{CoMCH}$.

The hospital incidence of PROM was found to be 1 in 16 (6.3\%). Among them, 114 (57.6\%) patients were admitted at term (>37weeks) and 84 (42.4\%) patients came before 37 completed weeks of gestation.

Table - I \& II shows that $41 \%$ of the affected women were primigravida and belonged to 20-24 years of age 107(54\%), were multigravida $117(59 \%)$. Ninety six (48.5\%) patients presented with different complications associated with PROM. Table III shows that most common complication of was subclinical urogenital infection (53\%) next oligohydramnios (25\%) $\&(13.5 \%)$ women presented with chorioamnionitis.

Most of the patients 153 (77.06\%) were delivered by caesarean section(C/S). Previous C/S, oligohydramnios, fetal distress, chorioamnionitis were the common indications for doing C/S (Table - III). Only $32(16.2 \%)$ patients were delivered vaginally, 8(4.24\%) patients responded to conservative management and $5(2.5 \%)$ patients were referred to tertiary centre for extreme prematurity with associated complicating factors (previous LUCS with bad obstetrical history). Table -IV shows most of the women delivered by LUCS (77.06\%). Only 8 women (4.24\%) could prolong their pregnancy.

Table $-V$ shows that most of the babies, $71(38.4 \%)$ had birth weight in between $2.1-2.5 \mathrm{~kg}, 26$ (14.1\%) babies had birth weight between 1.6 to $2 \mathrm{~kg}$ and 19 (10.3\%) babies were born with less than $1.5 \mathrm{~kg}$ body weight. Immediately after birth, 148(78.3\%) babies were born with Apgar score >7, 31(16.4\%) within 4-6, 9(4.76\%) babies had Apgar score $<3$. Only $1(0.53 \%)$ baby had no sign of life after birth (Table $-\mathrm{V})$. There was no maternal mortality but perinatal death was $5(2.5 \%)$.

Table-I

Age distribution of women of PROM ( $n=198)$

\begin{tabular}{lcc}
\hline Age group & Number of patients & Percentage $(\%)$ \\
\hline $15-19$ & 7 & 3.5 \\
$20-24$ & 107 & 54 \\
$25-29$ & 58 & 29.3 \\
$30-34$ & 20 & 10.1 \\
$>35$ & 6 & 3.0 \\
\hline
\end{tabular}

In this study, the age of the patients ranged between $15->35$ years. Most of the patients belonged to the age group $20-24$ years (54\%).

Table-II

Gravidity of the women of PROM ( $n=198)$

\begin{tabular}{lcc}
\hline Gravida & Number of patients & Percentage (\%) \\
\hline 1st & 81 & 40.9 \\
2nd & 57 & 28.8 \\
3rd & 29 & 14.6 \\
4th & 17 & 8.6 \\
5 th & 10 & 5.1 \\
$6^{\text {th }}$ & 3 & 1.5 \\
$7^{\text {th }}$ & 1 & 0.5 \\
\hline
\end{tabular}

Table II shows that $40.9 \%$ of PROM patients were primigravida and $59.1 \%$ of patients were multigravida.

Table-III

Complications of PROM

\begin{tabular}{lcc}
\hline Indication & $\begin{array}{c}\text { Number of } \\
\text { patients }\end{array}$ & Percentage (\%) \\
\hline Pregnancy with subclinical & 51 & 53 \\
urogenital infection & & \\
Oligohydramnios & 24 & 25 \\
Choioamnionitis & 13 & 13.5 \\
Pregnancy with Medical & 7 & 7.3 \\
disease & & \\
Pregnancy with appendicitis & 1 & 1 \\
\hline
\end{tabular}

Table III shows that most common complication of was subclinical urogenital infection (53\%) next oligohydramnios (25\%) \& (13.5\%) women presented with chorioamnionitis. 
Table-IV

Delivery outcome $(n=189)$

\begin{tabular}{lcc}
\hline Type of Delivery & $\begin{array}{c}\text { Number of } \\
\text { babies }\end{array}$ & Percentage (\%) \\
\hline LUCS & 153 & 77.06 \\
NVD & 32 & 16.2 \\
Not delivered & 8 & 4.24 \\
Referred & 5 & 2.5 \\
\hline
\end{tabular}

Table IV shows most of the women delivered by LUCS (77.06\%). Only 8 women (4.24\%) could prolong their pregnancy.

Table-V

Fetal Birth weight $(n=189)$

\begin{tabular}{lcc}
\hline Fetal weight $(\mathrm{Kg})$ & $\begin{array}{c}\text { Number of } \\
\text { babies }\end{array}$ & Percentage (\%) \\
\hline$<1.5$ & 19 & 10.3 \\
$1.6-2$ & 26 & 14.1 \\
$2.1-2.5$ & 71 & 38.4 \\
$2.6-3$ & 56 & 30.3 \\
$3.1-3.5$ & 13 & 7.02 \\
$>3.5$ & 4 & 2.11 \\
\hline
\end{tabular}

Table $V$ shows most of the babies $71(38.4 \%)$ had birth weight in between $2.1-2.5 \mathrm{~kg}$ and $19(10.3 \%)$ babies were less than $1.5 \mathrm{~kg}$ body weight.

Table-VI

Apgar Score of Babies ( $n=189)$

\begin{tabular}{lcc}
\hline Apgar Score & Number of babies & Percentage (\%) \\
\hline$>7$ & 148 & 78.3 \\
$4-6$ & 31 & 16.4 \\
$<3$ & 9 & 4.76 \\
0 & 1 & 0.53 \\
\hline
\end{tabular}

Maximum number of babies (78.3\%) had apgar score $>7$ and only $9(4.76 \%)$ babies born with apgar score $<3$.

\section{Discussion:}

To give birth a healthy baby is the most awaited event in a woman's life. PROM is an important cause of preterm labour and delivery of a low birth weight baby resulting in high perinatal mortality and morbidity ${ }^{3}$.

In our country, true incidence of PROM is very difficult to ascertain because more than $80 \%$ deliveries occur at home. This study, conducted at $\mathrm{CoMCH}$, shows the incidence of PROM in hospital was 6.3\%. A study undertaken at Dhaka Medical College Hospital $(\mathrm{DMCH})$ in 1995 showed the incidence of PROM to be $8.12 \%{ }^{7}$ and in 2001 it was $9.05 \%{ }^{8}$. Another study at Rangpur Medical College Hospital reported an incidence of $9.04 \%{ }^{9}$. The incidence at Indian studies from Mumbai reported by Bhalerao \& Desai and Bhide in 2000 \& 2001 showed an incidence of PROM between 7 and $12 \%^{10}$. Daftary and Desai in 2006 correlated the incidence of PROM with the gestational maturity and reported that PROM before the onset of true labour occured in 5-20\% of all women in labour ${ }^{11}$. Although there is some morbidity when PROM occurs in term pregnancies, the fundamental clinical problem is preterm PROM, a condition that occurs in $3 \%$ of all pregnancies and is responsible for approximately $30 \%$ of all preterm deliveries as reported by Arias and Tomich on $1982^{12}$.

During the study period, $57.6 \%$ patients were admitted at term PROM (>37weeks) and $42.4 \%$ patients came prior to 37 weeks (PPROM). In this study, most of the affected patients belongs to 20 -24 years of age (54\%), which is similar to other studies ${ }^{7-9}$. In many studies, high parity is one of the risk factor for increasing $\mathrm{PROM}^{7-9}$. In present study, maximum women were multigravida (59\%) coming from low socio-economic families. Low socio-economic status is an important risk factor for both PROM and preterm labour. Associated factors such as malnutrition, overexertion, poor hygiene, stress, recurrent genitourinary infections and anaemia considerably increase the risk ${ }^{1}$. In a study by Begum, $50 \%$ patients were in the group of low socioeconomic condition having no or irregular antenatal check-up which is almost similar to this study ${ }^{9}$.

In 1991, Romero et al reported that infection is twice as frequent in PROM than in preterm labour with intact membranes. In another study by Romero et al in 1993, in term PROM the incidence of infection was approximately $20 \%$ and in PPROM it was $38.3 \%{ }^{13}$. Also, women with PPROM and labor at the time of admission had a greater incidence of chorioamnionitis than women with PPROM admitted without labour ${ }^{13}$. Associated maternal medical and obstetrical complications had highly significant impact on PROM.

During the study period, $77.3 \%$ of PROM patients were admitted with different complications. Among them $8.5 \%$ patients presented with chorioamnionitis. About $54 \%$ patients had maternal complications and 
$11 \%$ patients had clinical evidence of choioamnionitis in the study by Begum ${ }^{9}$. Another study by Miller et al in 1978 and Johnson et al in 1981 observed prevalence of microbial invasion of amniotic cavity with PROM as $30 \% 14$.

In present study, high vaginal swab culture was positive in $25.8 \%$ patients. E.coli was most common pathogen. Because of limitation of facilities, anaerobic culture and other relevant investigations for diagnosis of subclinical or clinical amniotic fluid infection were not done, only aerobic culture was done. The evidence implicating infection as an etiologic agent in membrane weakening and rupture is robust. Between 14.6 and $38 \%$ of women with PROM will have positive amniotic fluid cultures ${ }^{2}$. The microorganisms isolated from amniotic fluid cultures (Peptostreptococcus, Bacteroides, Fusobacterium, Lactobacillus, Ureaplasma urealyticum) are similar to those normally found in the vagina, strongly suggesting that the source of infection is bacteria normally present in the vagina or the cervix. The mechanism of ascending infection is not clear ${ }^{2}$. Hein et al in 2001 observed that changes in the antibacterial properties of cervical mucus may also play an important role in facilitating ascending infection ${ }^{15}$. The importance of factors that theoretically may increase the possibility of ascending infection such as sexual intercourse, pelvic digital examinations during pregnancy and history of prior cervical dilatation for abortion or D\&C has been disproved by clinical investigations. On the other hand, colonization of the lower genital tract by Chlamydia, Neisseria gonorrhoeae, group B streptococcus, Tricomonas, E.coli and bacteroid species has been shown to increase the risk of $\mathrm{PROM}^{2}$.

In this series, $77.2 \%$ patients were delivered by caesarean section \& $16.2 \%$ patients were delivered vaginally. But Begum shows that only $32 \%$ patients were delivered by $\mathrm{C} / \mathrm{S}^{9}$. Puerperal sepsis $(5.6 \%)$ \& wound infections (21.25\%) were common puerperal complications which are similar to other studies ${ }^{7-9}$. A reduction in incidence of puerperal sepsis is due to proper use of antibiotics and timely termination of pregnancy ${ }^{9}$.

During our study period, most of the babies $38.4 \%$ born belonged to birth weight between $2.1-2.5 \mathrm{~kg}$, $14.1 \%$ babies were in between 1.6 to $2 \mathrm{~kg}$ and $10.3 \%$ babies were born with less than $1.5 \mathrm{~kg}$ body weight. Several studies have concluded that hyaline membrane disease is the greatest threat to the newborn when PROM occurs before term. The data from Mercer in 2003 show that at all gestational age the risk of respiratory distress is greater than the risk of infection. At 24 weeks $100 \%$ of the newborns develop respiratory distress syndrome, at 32 weeks $25 \%$ and at 34 weeks close to $10 \%$. The risk of sepsis at these gestational ages was $40,32,4.5$ and $3 \%$, respectively ${ }^{16}$.

In this study, all new born surveillance was not possible because of limitation of facilities. All new born with complications like asphyxia, sepsis, low birth weights were sent to neonatology ward for further management. Perinatal death was $2.5 \%$ in this study but there was no maternal mortality.

\section{Conclusion:}

Most of the affected women belongs to 20-24 years of age $107(54 \%)$, about $41 \%$ of them were primigravida and $117(59 \%)$ were multigravida. Term PROM was more in comparison to PPROM and most of them were multigravida. Caesarean section rate was high. Most common complication of was subclinical urogenital infection (53\%) next oligohydramnios (25\%) \& (13.5\%) women presented with chorioamnionitis. About $2 / 3$ of the babies birth weight were with in 2 to $2.5 \mathrm{~kg}$.

\section{Reference :}

1. Usha R. Krishna and Monisa H.Shah. Prelabour Rupture of Membranes, Obstetrics and Gynecology for Postgraduates, edited by S.S. Ratnam, K. Bhasker Rao and S. Arulkumaran, $2^{\text {nd }}$ edition-1999; Vol-1, 96-108.

2. Arias F, Daftary SN, Bhide AG. Premature Rupture of Membrane, Practical Guide to HighRisk Pregnancy \& Delivery , A South Asian Perspective, $3^{\text {rd }}$ edition-2008; 240-261.

3. D.C. Dutta. Prelabour Rupture of the Membranes, Text Book of Obstetrics, $6^{\text {th }}$ edition 2006, 317-320.

4. Pressman EK, Cavanaugh JL, Woods JR, et al. Physical properties of the chrioamnion throughout gestation. Am J Obstet Gynecol 2002; 187; 672-5.

5. Barabas AP. Ehlers-Danlos syndrome associated with prematurity and premature rupture of fetal membranes; possible increase in incidence. Br Med J 1966; 2: 682-4.

6. McGregor JA. French JL, Lawellin D, et al. Bacterial protease-induced reduction of 
choioamnionitic membrane strength and elasticity. Obstet Gynecol 1987; 69: 167-74.

7. Tasnim S: Clinical Profile and Outcome of Pregnancy in Premature Rupture of Membrane -A Study in Dhaka Medical College Hospital. Dissertation; Bangladesh College of Physicians and Surgeons, 1995.

8. Begum A ; Maternal and Fetal Outcome of Premature Rupture of Membrane -One year Study in Dhaka Medical College Hospital. Dissertation; Bangladesh College of Physicians and Surgeons, 2001.

9. Begum N; Epidemiology of Premature Rupture of Membrane and Management in Rangpur Medical College Hospital. Dissertation; Bangladesh College of Physicians and Surgeons, 2004.

10. Bhalerao $S$, Desai $A$. Premature rupture of membranes. Jn: Saraiya UB, Rao KB, Chatterjee $A$, eds. Principles and Practice of Obstetrics\& Gynecology (2 ${ }^{\text {nd }}$ edn). An FOGSI Publication. New Delhi: Jaypee Brothers, 2003: 125.

11. Daftary SN, Desai SV. Preterm labour and premature rupture of membranes. In: Daftary SN,
Desai SV, eds. Selected Topics in Obstetrics \& Gynaecology (2 ${ }^{\text {nd }}$ edn). New Delhi: BI Publications, 2006: 128.

12. Arias F, Tomich PH. Etiology and outcome of low birth weight and preterm infants. Obstet Gynecol 1982; 60: 277-81.

13. Romero R, Yoon $\mathrm{BH}$, Mazor $\mathrm{M}$, et al. $\mathrm{A}$ comparative study of the diagnostic performance of amniotic fluid glucose, white cell count, interleukin-6 and Gram stain in the detection of microbial invasion in patients with preterm premature rupture of the membranes. Am J Obstet Gynecol 1993; 169: 839-51.

14. Johnson J WC, Daikoku NH, Niebyl JR et al. Premature rupture of membranes and prolonged latency. Obstet Gynecol 1981; 57: 547-556.

15. Hein M, Helmig RB, Schonheyder HC, et al. An in vitro study of antibacterial properties of the cervical mucous plug in pregnancy. Am J Obstet Gynecol 2001; 185.

16. Mercer BM. Preterm premature rupture of the membranes. Obstet Gynecol 2003; 101: 178-93. 